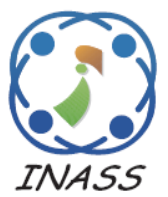

\title{
Power Spectral Density of V2V Communication System in the Presence of Moving Scatterers
}

\author{
Wahyu Pamungkas ${ }^{1,2 *}$ \\ Titiek Suryani ${ }^{1}$ \\ Wirawan Wirawan ${ }^{1}$ \\ Achmad Affandi ${ }^{1}$ \\ ${ }^{l}$ Department of Electrical Engineering, Faculty of Electrical Technology, \\ Institut Teknologi Sepuluh Nopember, Surabaya, Indonesia \\ ${ }^{2}$ Fakultas Teknik Telekomunikasi dan Elektro, Institut Teknologi Telkom, Purwokerto, Indonesia \\ * Corresponding author's Email: wahyu @ittelkom-pwt.ac.id
}

\begin{abstract}
A communication system of Vehicle to Vehicle (V2V) consists of a transmitter, a receiver, and scatterers which can move in random speeds and directions to generate the Doppler Effect that reduces communications system performance. The Doppler effect that occurs in V2V channel modeling is a combination of the Doppler effect on each of its constituent components. After being combined with the Orthogonal Frequency Division Multiplexing (OFDM) multi-carrier system, the signal received at the receiving end experiences attenuation due to the characteristics of the channel used. To analyze the attenuation factor received due to the influence of channel characteristics, the power spectral density parameter is used. Previous research was limited to modeling the V2V channel with moving scatterers, without knowing the effect of velocity on power spectral density. The purpose of this study was to examine the effects of velocity parameters of V2V's components on power spectral density where scatterers moved randomly. Moving scatterers speed was classified into two categories, where the scatterers were faster or slower than the sender and receiver. The power spectral density was analyzed after combining Quadrature Phase Shift Keying (QPSK) modulation with multi-carrier OFDM. The results show that components with higher velocity yielded more damped power spectral density on the receiver that reached $-0.7099 \mathrm{~dB} / \mathrm{Hz}$. Moreover, the number of scatterers that moved around the transmitter and receiver highly affected the magnitude of the Doppler effect. The largest Doppler Shift generated in this study was $13.5 \mathrm{kHz}$ with attenuated power spectral density at $-0.8646 \mathrm{~dB} / \mathrm{Hz}$.
\end{abstract}

Keywords: Power spectral density, V2V channel, Moving scatterer, Doppler effect, QPSK modulation, OFDM.

\section{Introduction}

Current wireless communication technologies not only serve users through cellular, satellite, radar or microwave services but also through vehicles that can communicate with each other. The vehicle-based communication system provides a variety of services ranging from vehicle communications without a driver to the internet connection between vehicles that are ubiquitous. This service is required to provide a sense of comfort and security to all users [1].

Standard communication systems between vehicles and other vehicles have been developed in recent years to regulate interoperability of information exchange between vehicles. Among these standards are Dedicated Short Range Communications (DSRC) developed in the United States, and the standard of Intelligent Transport System (ITS) developed by the European Telecommunications Standard Institute (ETSI) [2]. Both standards are developed based on IEEE 802.11p technology as a standard for Vehicle Ad Hoc Network (VANET) [3]. Furthermore, on the mapping of 7 OSI layers, DSRC technology is placed on the Physical and Data Link layers and the rest is used for the IEEE 1609 Wireless Access Vehicle (WAVE) standard [4].

One communication model in VANET technology is Vehicle to Vehicle (V2V), which is used to accommodate the connection between a vehicle and other vehicles [5]. The characteristic of 
V2V differs from Fix to Mobile (F2M) and Mobile to Mobile (M2M) communication model in the radio communication that has been developed. In the F2M communication model, users on the mobile station side are considered moving, however, users on the base station are considered static. Meanwhile, in the V2V communication system, users on the transmitter and receiver are considered randomly moving, equipped with a low elevated antenna, surrounded by scatterers that are also moving randomly [6]. Another characteristic of $\mathrm{V} 2 \mathrm{~V}$ communication model is the influence of the environment around the communication system and the traffic density [7].

M2M communication model that considers the movement of the transmitter, receiver, and scatterers were introduced by [8]. This modeling provides statistical analysis by calculating parameters such as probability distribution of received signal envelope, spatial time correlation function, and power spectral density of the received signal envelope. Further development of this model is conducted by involving second-order statistic including crossing rate level, fade duration, the random probability distribution of FM, expected random number of crossing from a phase, and power spectrum. Power spectrum explained the biggest power distribution in a certain bandwidth range [9].

A different M2M communication system model is developed by initially placing scatterers on the circle surrounding the transmitter and receiver. This concept is called a correlated double-ring [10]. In this model, the number of scatterers on the transmitter and receiver are assumed to be the same while the velocity of the transmitter and receiver are assumed to be the same but with random directions. This modeling is validated by using autocorrelation function and probability density function for Rayleigh and Rician distribution. For Rayleigh fading channel for the wireless communication system, it has been proved that for flat and frequency selective fading channel condition, the performance of the Bit Error Rate (BER) parameter improved in the value of the signal to noise ratio (SNR), which is equal to $45 \mathrm{~dB}$ [11].

The correlated double-ring model used for $\mathrm{V} 2 \mathrm{~V}$ application was developed by [12] by accommodating the movement of a transmitter, receiver, and scatterers that are placed on the radius of a circle surrounding the transmitter and receiver. This model required wave propagation from the transmitter to surrounding scatterers towards scatterers on the receiver side then goes to the receiver. This scheme is known as a single bounce. Results indicate that near and far moving scatterers yielded different effects in fading channel statistic.
In a different model, V2V modeling using geometrical street model is defined by [13], encompassing moving and fix scatterers in the highway propagation environment. In [14], this model is developed using a three-dimensional (3D) geometrical propagation model that includes line-ofsight (LOS), single bounced (SB), and multiple bounced (MB) rays. In subsequent modeling, [15] developed the V2V model by emphasizing the Doppler effect due to moving scatterers. This modeling is also equipped with analysis using autocorrelation parameters and spectral power components [16]. The integral value of the power spectral density parameters in the entire frequency range is equal to the average power [17].

Moreover, in multi-carrier technology OFDM with high-efficiency amplification, modeling power spectral density has been done using 802.11a. Research on Doppler power spectral density by accommodating the presence of roadside scatterers has been carried out. Roadside scatterers are assumed to be on both sides of the highway. The modeling simulation results are then compared with the measurement method to be validated [18].

As is known, there are two methods in determining the parameters of power spectral density[17]. The first method uses the decomposition of fourier transforms of signals containing random processes. The second method is to perform a fourier transformation of the autocorrelation function of the signal which contains a random process. Research on the modeling of power spectral density on the V2V channel with a mobile scatterer has also been carried out with a 3-D model under Line of Sight (LOS) conditions, single bounced and double bounced rays [19]. This research uses determining power spectral density using a method through the fourier transform of the autocorrelation function. However, in this study, the modeling results had not been combined with the multicarrier system used in VANET standardization.

Some key contributions of this paper are as follows :

1. We used the decomposition of the Fourier transform of a signal which comprising the random process.

2. Integrating $\mathrm{V} 2 \mathrm{~V}$ channel model with moving scatterer with multi-carrier OFDM and use QPSK modulator to transmit the signal with single bounced model. The communications system was modeled based on the block diagram as seen in Fig 2. The complex envelope of the $\mathrm{V} 2 \mathrm{~V}$ channel was processed with channel impulse response and convoluted with output IFFT on OFDM and Additive White Gaussian 
Noise (AWGN) was added. As far as we know, no paper has ever used $\mathrm{V} 2 \mathrm{~V}$ channel with moving scatterer and integrating with multi-carrier OFDM.

3. Using the mobility of scatterer to get power spectral density with the velocity of the scatterer that was more dominant than the speed of the transmitter's and receiver's vehicles. This configuration generated a unique power spectral density parameter compared to the dominance of vehicle speed as the transmitter and receiver.

The next sections of this paper will be divided as follows. Section II discusses the research method and the channel modeling characteristics using the V2V Channel in the presence of moving scatterer and the concept of combining the channel with OFDM. Meanwhile, Section III presents the results and analysis, and Section IV presents the conclusions.

\section{Research method}

\section{$2.1 \mathrm{~V} 2 \mathrm{v}$ channel model in the presence of moving scatterer}

Wave propagation modeling in $\mathrm{V} 2 \mathrm{~V}$ communication with the transmitter, receiver, and moving scatterers is depicted in Fig. 1. Transmitter $\left(T_{x}\right)$ and receiver $\left(R_{x}\right)$ are surrounded by $\mathrm{N}$ scatterers denoted with $\mathrm{S}_{\mathrm{n}}(\mathrm{n}=1,2,3, \ldots, \mathrm{N})$. The velocity of $T_{x}$ and $R_{x}$ are assumed constant, which are denoted with $V_{T}$ and $V_{R}$ and their directions are defined by angles $\alpha_{v}^{T}$ and $\alpha_{v}^{R}$.

Whereas each of randomly moving scatterers denoted with $v_{s_{n}}$ and their directions are denoted with $\alpha_{v}^{s_{n}}$. In this modeling, owing to the high path loss, the scatterers around the transmitter and receiver that located at a remote are ignored and assumed that wave propagated from transmitter with Angle of Departure (AoD) $\alpha_{n}^{T}$ to receiver with Angle of Arrival (AoA) $\alpha_{n}^{R}$ immediately after it bounces once on scatterers inside the propagation area.

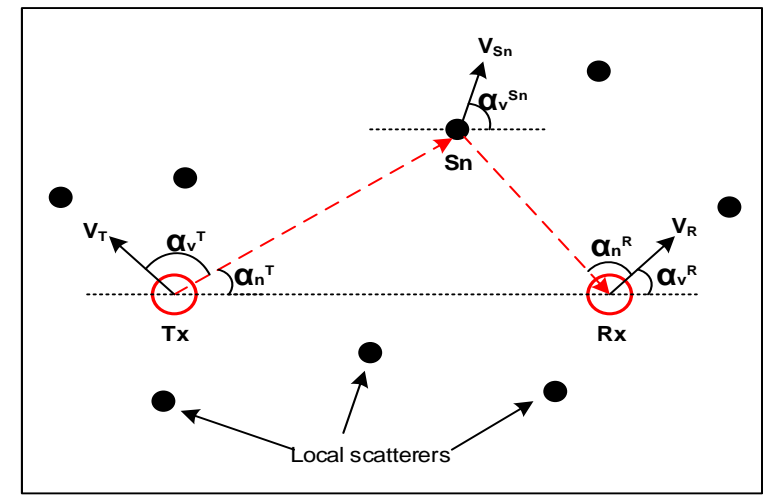

Figure. 1 Propagation scenario in V2V communication model
To determine the parameter of the complex channel gain of channel modeling being used, an assumption from frequency modeling of nonselective channel Fix to Vehicle $(\mathrm{F} 2 \mathrm{~V})$ is used. This modeling required that $T_{x}$ is static, but $R_{x}$ can move with high velocity and surrounded by static scatterers. This system is modeled as follows [15].

$$
\mu(t)=\sum_{n=1}^{N} c_{n} e^{j\left(2 \pi f_{n} t+\theta_{n}\right)}
$$

The attenuation factor is denoted with $c_{n}$ and $f_{n}$ is the Doppler frequency as a result of receiver movement. All the path gain $c_{n}$ have the same size with value $c_{n}=\sigma_{0} \sqrt{2 / N}$. The parameter $\theta_{n}$ is a phase shift from nth propagation that uniformly distributed in the range of $0-2 \pi$. This channel gain is a mixture of all incoming path line towards receiver after they bounced on scatterers.

In the V2V channel modeling, there is a fundamental difference to the F2V channel modeling. This fundamental difference is that the transmitter and receiver are moving simultaneously and both of them are surrounded by scatterers that are also moving. As a consequence, the Doppler effect as a result of the movement of the transmitter, receiver, and their scatterers are taken into account. Hence, $f_{n}$ in the eq (1) is replaced with the following.

$$
f_{n}=f_{n}^{T}+f_{n}^{T s}+f_{n}^{S R}+f_{n}^{R}
$$

The parameter denoted with $f_{n}^{T}$ is the Doppler effect caused by transmitter movement. The parameter $f_{n}^{T S}$ is the parameter caused by a transmitted signal that hit $n$-th moving scatterers. The parameter $f_{n}^{S R}$ is the effects of scatterers that move and bounce signals towards the receiver. The parameter $f_{n}^{R}$ is the Doppler caused by receiver movement. Each of these Doppler frequency components in eq (2) can be defined as follows.

$$
\begin{aligned}
f_{n}^{T} & =f_{0} \frac{V_{T}}{C_{0}} \cos \left(\alpha_{v}^{T}-\alpha_{n}^{T}\right) \\
f_{n}^{T_{S}} & =\left(f_{0}+f_{n}^{T}\right) \frac{v_{s_{n}}}{c_{0}} \cos \left(\pi+\alpha_{n}^{T}+\alpha_{v}^{s_{n}}\right) \\
& \approx-f_{0} \frac{v_{S_{n}}}{c_{0}} \cos \left(\alpha_{n}^{T}-\alpha_{v}^{s_{n}}\right) \\
f_{n}^{S_{R}} & =\left(f_{0}+f_{n}^{T_{S}}\right) \frac{v_{S_{n}}}{c_{0}} \cos \left(\pi+\alpha_{v}^{S_{n}}+\alpha_{n}^{R}\right) \\
& \approx-f_{0} \frac{v_{s_{n}}}{c_{0}} \cos \left(\alpha_{v}^{S_{n}}-\alpha_{n}^{R}\right)
\end{aligned}
$$




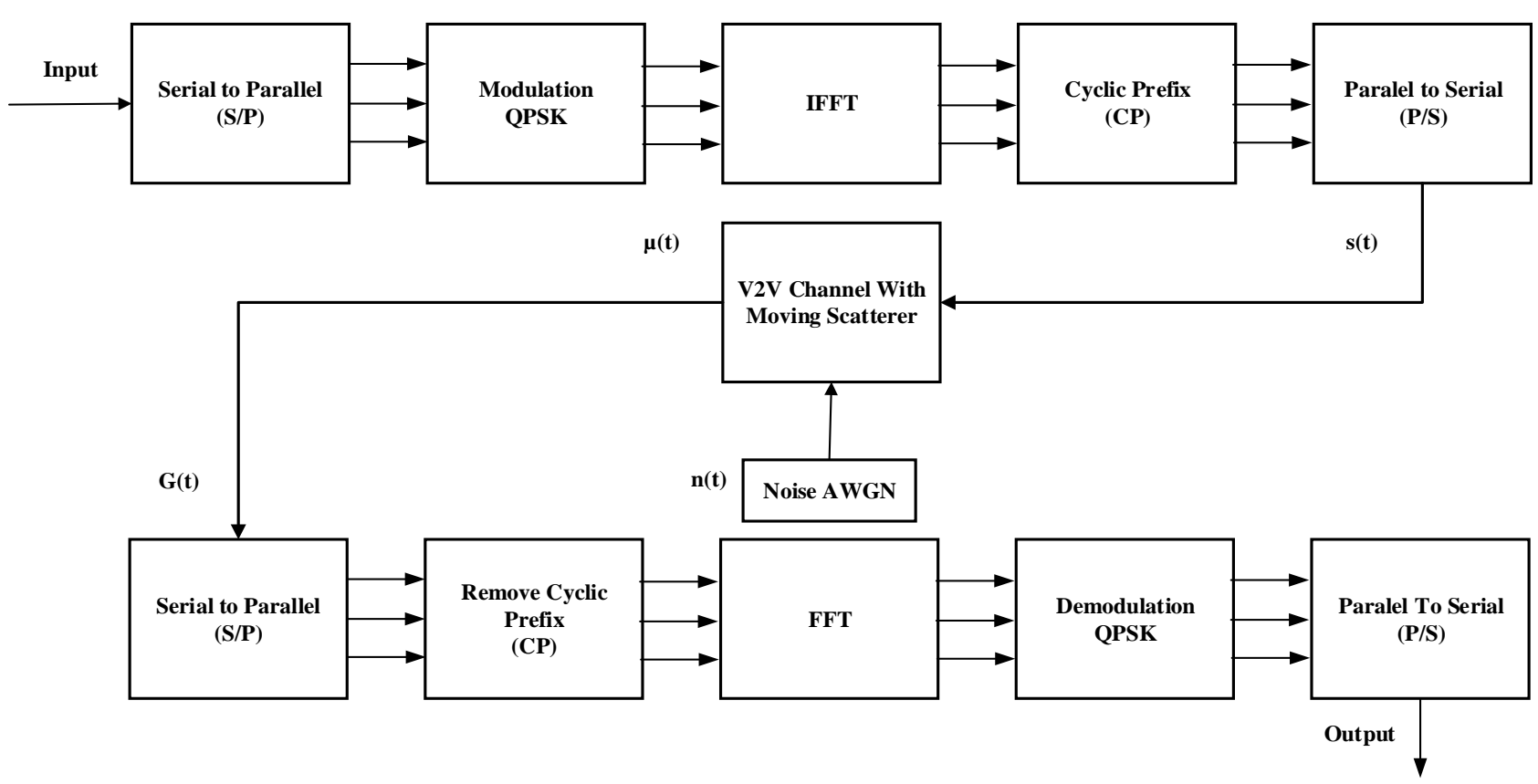

Figure.2 Model of V2V channel system with OFDM

$$
\begin{aligned}
f_{n}^{R} & =\left(f_{0}+f_{n}^{S_{R}}\right) \frac{v_{\mathrm{R}}}{c_{0}} \cos \left(\alpha_{v}^{R}-\alpha_{n}^{R}\right) \\
& \approx f_{0} \frac{v_{R}}{c_{0}} \cos \left(\alpha_{v}^{R}-\alpha_{n}^{R}\right)
\end{aligned}
$$

However, these Doppler frequency values are small and less than f0 which has a value of $5.8 \mathrm{GHz}$. $\mathrm{Eq}$ (3) to (6) to calculate each of Doppler frequency individually can be summarized into one formula denoted with fn as follows [15].

$$
\begin{gathered}
f_{n}=\frac{k_{0}}{2 \pi}\left[v_{T} \cos \left(\alpha_{v}^{R}-\alpha_{n}^{T}\right)-v_{S_{n}}+\left(\operatorname { c o s } \left(\alpha_{n}^{T}-\right.\right.\right. \\
\left.\left.\left.\alpha_{v}^{S_{n}}\right)+\cos \left(\alpha_{v}^{S_{n}}-\alpha_{n}^{R}\right)\right)+v_{T} \cos \left(\alpha_{v}^{R}-\alpha_{n}^{R}\right)\right]
\end{gathered}
$$

with the parameter $k_{0}=2 \pi \frac{f_{0}}{c_{0}}$ is a free space wavenumber.

\subsection{Multi-carrier OFDM}

VANET has a bandwidth of $10 \mathrm{MHz}$. This bandwidth in the vehicular environment is susceptible to multipath fading and users that move with high velocity. Moreover, channel bandwidth suffers from doubly selective in frequency domain and time domain. Channel response in one period has fluctuated power strength and delayed propagation that cause Inter-Symbol Interference (ISI). Furthermore, the frequency response of the channel has different characteristics and fluctuation that cause Inter-Carrier Interference (ICI) phenomenon.
Based on the phenomenon, the multi-carrier system implemented on the VANET service is Orthogonal Frequency Division Multiplexing (OFDM) [25]. In this paper, QPSK modulation with Cyclic Prefix (CP), and conversion process of Serial to Parallel (S/P) and Parallel to Serial (P/S) were used. QPSK modulation used 4 subcarriers that transmit a 64-bit number of data. Therefore, the comparison between technology 802.11a and 802.11p on the physical layer become as in Table 1 .

In multi-carrier OFDM, the IFFT output signal that will be integrated is formulated as follows.

$$
s(t)=\sum_{k=0}^{N=1} X_{x} \exp (-j 2 \pi k \Delta f t)
$$

Table 1. Physical layer differences between 802.11p and $802.11 \mathrm{a}[20]$

\begin{tabular}{|l|l|l|}
\hline Parameter & $802.11 \mathrm{p}$ & $802.11 \mathrm{a}$ \\
\hline Band Frequency & $\begin{array}{l}5.85-5.95 \\
\mathrm{GHz}\end{array}$ & $\begin{array}{l}5.15-5.35 \\
\mathrm{GHz}\end{array}$ \\
\hline Data Rate & $\mathrm{Max} 27 \mathrm{Mbps}$ & $\mathrm{Max} 54 \mathrm{Mbps}$ \\
\hline $\begin{array}{l}\text { Channel } \\
\text { Bandwidth }\end{array}$ & $10 \mathrm{MHz}$ & $20 \mathrm{MHz}$ \\
\hline $\begin{array}{l}\text { Number of } \\
\text { Channel Signal }\end{array}$ & $7 \mu \mathrm{s}$ & 12 \\
\hline $\begin{array}{l}\text { OFDM } \\
\text { Duration }\end{array}$ & $1.6 \mu \mathrm{s}$ & $0.8 \mu \mathrm{s}$ \\
\hline Guard Time & $6.4 \mu \mathrm{s}$ & $3.2 \mu \mathrm{s}$ \\
\hline FFT Period & $32 \mu \mathrm{s}$ & $16 \mu \mathrm{s}$ \\
\hline $\begin{array}{l}\text { Preamble } \\
\text { Duration }\end{array}$ &
\end{tabular}


With $\mathrm{N}$ is the number of IFFT point, $X k$ is the symbol data, and $\mathrm{f}$ is frequency.

\subsection{Integrating V2V channel model with multi- carrier OFDM}

The modeled channel will be integrated with a multi-carrier system which is used in VANET. After the transmission block, the signal is combined with Additive White Gaussian Noise (AWGN) and spreading out into the $\mathrm{V} 2 \mathrm{~V}$ channel that considers moving scatterers. Block diagram of this system model that incorporates channel modeling with OFDM multi-carrier is shown in Fig. 2.

The first step taken to integrate V2V Channel with multi-carrier OFDM is to express the channel gain in $\mathrm{Eq} 1$ as a complex number.

$$
Z(t)=x(t)+j y(t)
$$

Parameter $x(t)$ is the real value and parameter $y(t)$ describes the imaginer value. The absolute value of a complex envelope $Z(t)$ can be calculated as follows.

$$
R(t)=|Z(t)|=\sqrt{x(t)^{2}+y(t)^{2}}
$$

To calculate the value of the phase angle, the following formula can be used.

$$
\theta_{t}=\tan ^{-1}\left(\frac{y(t)}{x(t)}\right)
$$

Therefore, real part, imaginary part, and its absolute value can be expressed as follows.

$$
\begin{aligned}
& \mathrm{x}(\mathrm{t})=\operatorname{Re}\{Z(t)\}=R(t) \cos \left(\theta_{t}\right) \\
& \mathrm{y}(\mathrm{t})=\operatorname{Im}\{Z(t)\}=R(t) \sin \left(\theta_{t}\right)
\end{aligned}
$$

So, to get the rectangular version from the parameter $Z(t)$ we used:

$$
Z(t)=R(t) \cdot e^{j \theta_{t}}
$$

The second step is to establish the impulse response channel of the $\mathrm{V} 2 \mathrm{~V}$ channel. To calculate the value of the impulse response of the modeled channel, the following formula is used.

$$
h(t, \tau)=\sum_{K=1}^{N} R(t) \delta\left(\tau-\tau_{k}\right) e^{j \theta_{t}}
$$

With $\mathrm{N}$ is equal to 8 scatterers.

The third step is to combine the convolution between the impulse response channel $h(t)$ and the
IFFT output of OFDM $s(t)$ and add AWGN noise $n(t)$.

$$
G(t)=s(t) h(t)+n(t)
$$

The final step to find power spectral density, we used decomposition of fourier transform of signal which comprising the random process. Power spectral density of received signal $G(t)$ can be found as follows [17].

$$
S_{x}(f)=\lim _{T \rightarrow \infty} E\left\{\frac{1}{2 T}\left|\int_{-T}^{T} G(t) e^{-j 2 \pi f t} d t\right|^{2}\right\}
$$

\section{Result and discussion}

The simulation was conducted by modeling the effects of the velocity of the transmitter, receiver, and scatterers on the value of power spectral density. Simulation results were divided into 2 parts. The first part was the effect of the transmitter or receiver velocity that significantly increased while the velocity of moving scatterers was slightly increased. Meanwhile, the other part was the effect of the significantly increased velocity of moving scatterers while the velocity of the transmitter and receiver were slightly increased. On each scenario, the Doppler frequency $f_{n}$ was generated to be compared with power spectral density.

Predetermined parameters in this study were as follows.

- Carrier Frequency

$$
=5.8 \mathrm{GHz}
$$$$
\text { - } \text { Number of Scatterers } \quad=8
$$$$
\text { - } \alpha_{v}^{T} \text { and } \alpha_{v}^{R} \quad=15^{\circ}
$$$$
\text { - } \alpha_{v}^{R} \text { and } \alpha_{n}^{R} \quad \text { = random within }
$$$$
\text { - } \text { Bandwidth } \quad=40 \mathrm{MHz}
$$$$
\text { - } \text { Sampling Frequency } \quad=100 \mathrm{~Hz}
$$$$
\text { - } \text { Transmitted Data = } 64 \text { bit }
$$$$
\text { - Modulation = QPSK }
$$

- Number of Sub-carriers $=4$

\subsection{The effect of transmitter and receiver velocity on the power spectral density}

The transmitter and receiver velocity in this scenario were adjusted starting from low speed (15, 30 and $45 \mathrm{~km} / \mathrm{h}$ ), medium speed (50, 75, and 100 $\mathrm{km} / \mathrm{h})$ and high speed $(125,150$, and $175 \mathrm{~km} / \mathrm{h}$ ) while scatterers moved with speed slightly increasing from $3-5 \mathrm{~km} / \mathrm{h}$. Each scenario yielded the Doppler frequency $f_{n}$ value related to the power spectral density parameter in the range of frequency within the predetermined bandwidth. 


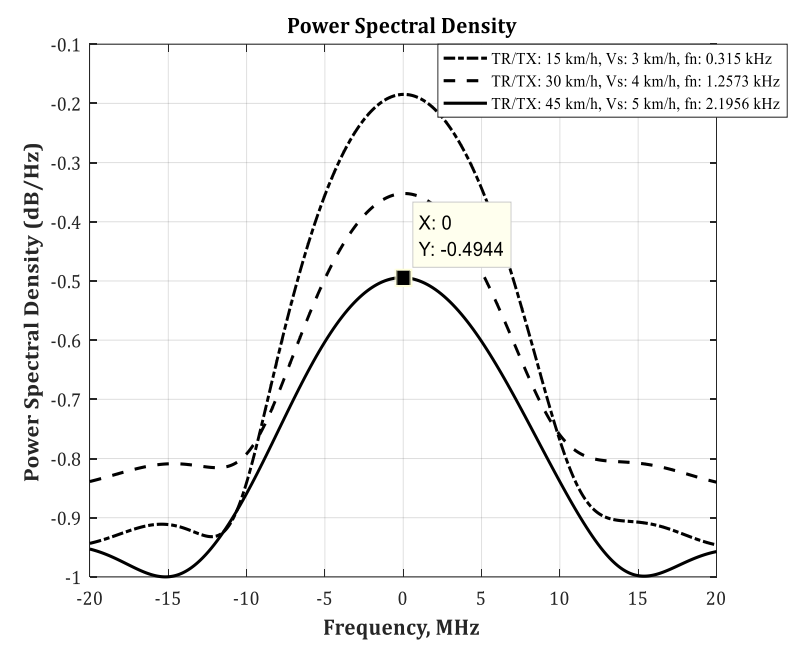

Figure. 1 The effect of transmitter/receiver velocity on the power spectral density at low speed

The simulation result displayed in Fig. 3 shows that the first iteration of the scenario yielded the smallest Doppler frequency of $315 \mathrm{~Hz}$ while the power spectral density was damped by approximately $-0.2 \mathrm{~dB} / \mathrm{Hz}$. Furthermore, when the transmitter and receiver velocity increased significantly while the moving scatterers velocity increased slightly, the generated Doppler frequency increase significantly.

This result was followed by a significant amount of attenuation on the power spectral density value up to $-0.4944 \mathrm{~dB} / \mathrm{Hz}$ for the Doppler frequency of $2.1956 \mathrm{kHz}$. In the wider frequency range, the value of power spectral density tends to decrease. This is because, in the wider frequency range, the value of the Doppler Effect becomes greater, which reduces the power spectral density value. The range of power spectral density from the lowest Doppler frequency to the largest at this scenario approximately reached $0.3 \mathrm{~dB} / \mathrm{Hz}$.

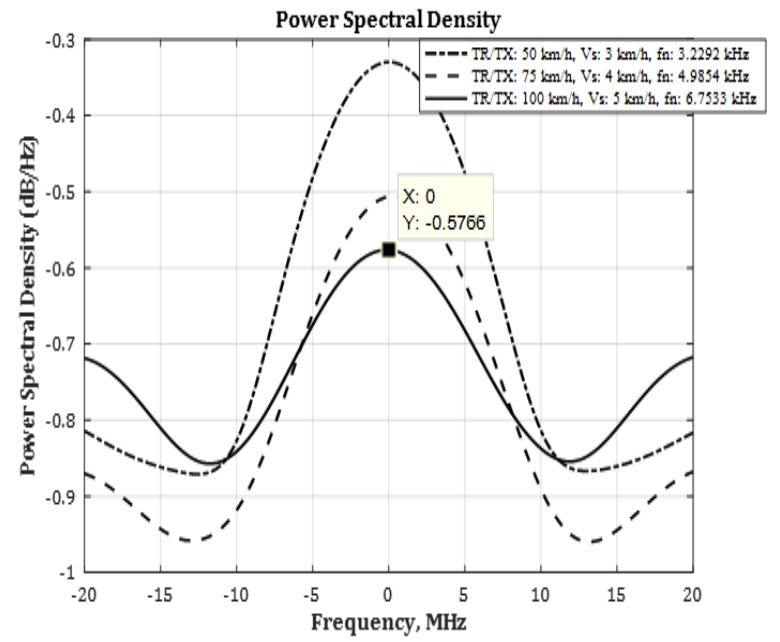

Figure. 2 The effect of transmitter/receiver's velocity on the power spectral density on medium speed

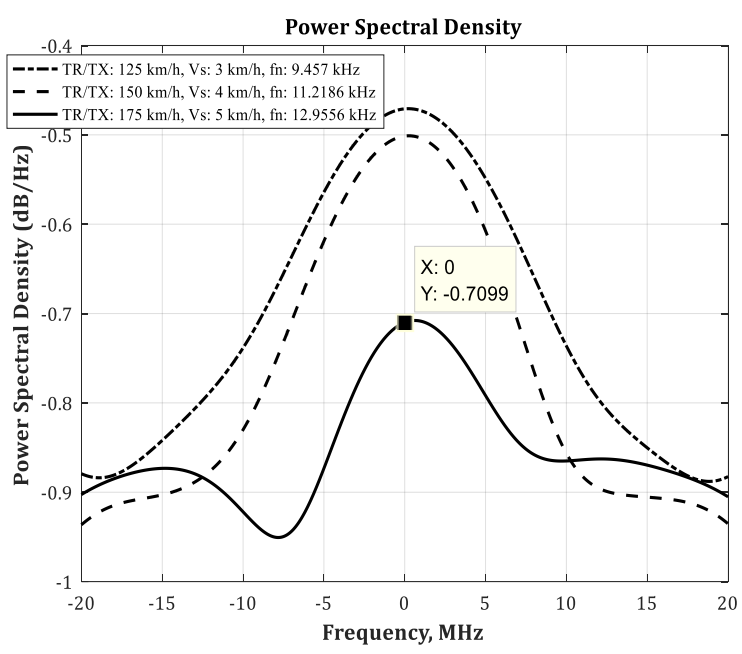

Figure. 3 The effect of transmitter/receiver velocity on the power spectral density at high speed

In the medium-speed scenario presented in Fig. 4, the increase in speed at the first iteration of $50 \mathrm{~km} / \mathrm{h}$ generated a Doppler frequency almost 10 times higher than the first iteration at a low speed of 15 $\mathrm{km} / \mathrm{h}$. The range of power spectral density from the lowest Doppler frequency to the largest at this scenario approximately reached $0.25 \mathrm{~dB} / \mathrm{Hz}$. Furthermore, at a speed of $100 \mathrm{~km} / \mathrm{h}$, the power spectral density damped up to $-0.5766 \mathrm{~dB} / \mathrm{Hz}$ for a Doppler frequency value of $6.7533 \mathrm{kHz}$.

In the third iteration, as shown in Fig. 5, the Doppler frequency as the result of the simulation reached a value above $6 \mathrm{kHz}$. The highest Doppler frequency was generated when transmitter and receiver moved with a speed of $175 \mathrm{~km} / \mathrm{h}$ while moving scatterers around them moved with a speed of $5 \mathrm{~km} / \mathrm{h}$. In this condition, the value of the power spectral density was $-0.7099 \mathrm{~dB} / \mathrm{Hz}$. The range of power spectral density from the lowest Doppler frequency to the largest at this scenario approximately reached $0.24 \mathrm{~dB} / \mathrm{Hz}$.

\subsection{The effect of dominant moving scatterers velocity on power spectral density}

In this section, two iterations were compared where the velocity of the transmitter and receiver were equal while moving scatterers had quite a significant increment of velocity. Theoretically, the Doppler frequency will be affected by referring to Eqs. (2) and (7). The Doppler frequency component in formula (2) was a combination of Doppler frequency which was induced by movement of the transmitter, receiver, and from the transmitter to its scatterers and also to the receiver from its scatterers. The number of scatterers used in this simulation was set to 8 . This component and the velocity of each 
scatterer gave the most significant impact on the value of Doppler frequency.

Velocities of moving scatterers were divided into two groups, which were scatterers that moved with speeds lower than speeds of transmitter and receiver and scatterers that moved with speeds higher than speeds of transmitter and receiver. The purpose of the division was to represent the real environment in the highway to simulate vehicles' velocity condition compared to moving scatterers around them. In the equal velocity of transmitter and receiver, while the velocity of moving scatterers was slightly different with the previous simulation in Fig. 3, the simulation gave higher Doppler frequency value as can be seen in Fig. 6.

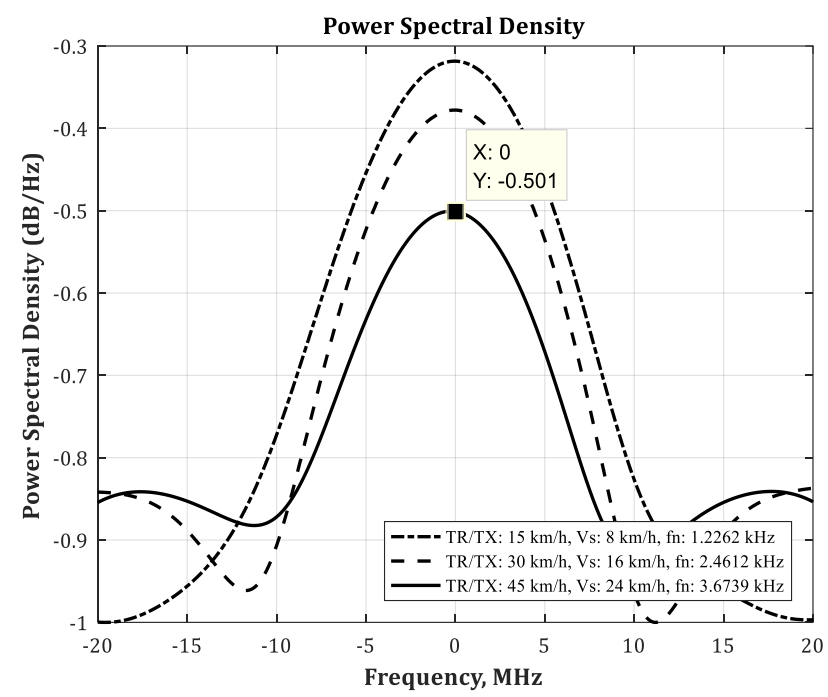

Figure. 4 The effects of moving scatterers that were slower than transmitter/receiver velocity on power spectral density

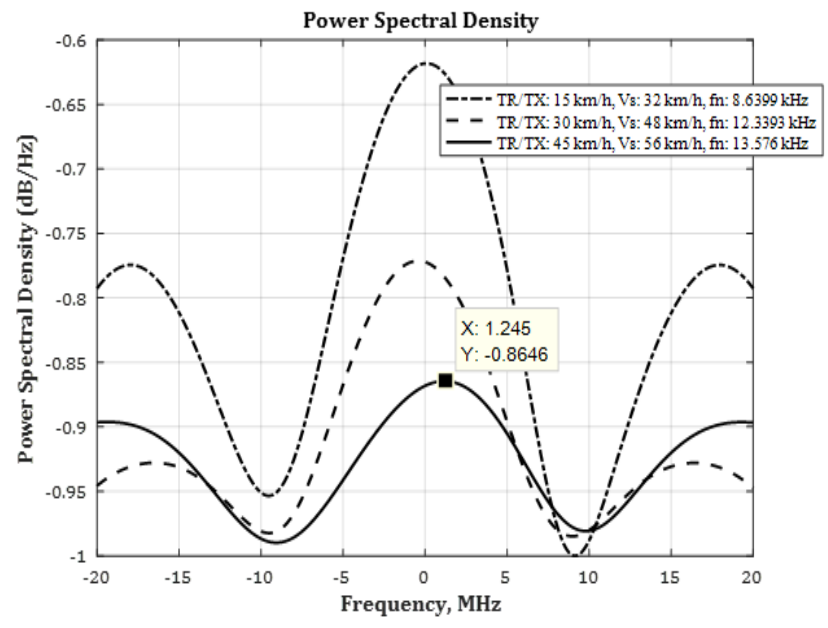

Figure. 7 The effects of moving scatterers that were faster than transmitter/receiver velocity on power spectral density
When the velocity of transmitter and receiver were at $45 \mathrm{~km} / \mathrm{h}$ and scatterers moved at $24 \mathrm{~km} / \mathrm{h}$, it resulted in the Doppler frequency of approximately $3.6 \mathrm{kHz}$ which was higher than the previous simulation. This can be seen in Fig. 3 on the third iteration while maintaining similar velocity pattern. The range of power spectral density from the lowest Doppler frequency to the highest at this scenario approximately reached $0.28 \mathrm{~dB} / \mathrm{Hz}$.

A second iteration where scatterers moved faster than the transmitter and receiver speeds generated a very large Doppler frequency value as seen at Eq (2) and (7). This is caused by the speed of each scatterer was combined with the speed of the other moving scatterers which were 8 scatterers. When the transmitter/receiver moved at a speed of $45 \mathrm{~km} / \mathrm{h}$ and scatterers moved at a speed of $56 \mathrm{~km} / \mathrm{h}$, it generated the Doppler frequency that reached $13.576 \mathrm{KHz}$. In this condition, the power spectral density parameter was damped up to - $0-8646 \mathrm{~dB} / \mathrm{Hz}$ as illustrated in Fig. 7. The range of power spectral density from the lowest Doppler frequency to the highest at this scenario approximately reached $0.24 \mathrm{~dB} / \mathrm{Hz}$.

\section{Conclusion}

This study analyzed the V2V channel modeling with moving scatterers that were combined with OFDM multi-carrier system and QPSK modulation. Simulation with various scenarios which was differentiated by the velocity of the transmitter, receiver, and moving scatterers shown that the faster they are moving, the higher Doppler frequency induced and the higher the attenuation of the power spectral density. In conclusion, power spectral density is attenuated on the receiver would be disadvantageous to a communication system. Therefore, careful and precise estimation of the transmitter, receiver, and moving scatterers' velocity is essential. This study also concludes that the number of scatterers and their velocity are parameters with the most influence on the attenuation of power spectral density according to channel modeling that was used. The scatterers generated a Doppler Shift of $13.5 \mathrm{kHz}$ with the power spectral density attenuated until $-0.8646 \mathrm{~dB} / \mathrm{Hz}$.

\subsection{Future works}

This study focused on power spectral density modeling due to the effect of the Doppler frequency on V2V channel modeling. Future research that will use the same $\mathrm{V} 2 \mathrm{~V}$ channel modeling to analyze the performance of Signal to Noise Ratio (SNR) and Bit Error Rate (BER) parameters in different modulation 
schemes with variations of transmitter / receiver speed and adjustable moving scatterers.

\section{Acknowledgments}

This research was supported by the Ten November Institute of Technology (ITS) through the Laboratory Research Grant 2019.

\section{References}

[1] S. J. Elias, S. M. Hatim, M. Y. Darus, S. Abdullah, T. Mara, and U. Kedah, "Congestion control in vehicular adhoc network: a survey", Indonesian Journal of Electrical Engineering and Computer Science, Vol. 13, No. 3, pp. 1280-1285, 2019.

[2] A. Chachich, V. Fessmann, J. Arnold, D. Thompson, W. Fehr, and S. Stasko, "DSRCUnlicensed Device Test plan To characterize the existing radio frequency signal environment", US Departement of Transportation, Washington DC, 2015.

[3] ETSI, "ETSI TR 101607 V1.1.1 - Intelligent Transport Systems (ITS); Cooperative ITS (CITS); Release 1", Route des Lucioles-France, 2013.

[4] C. Campolo and A. Molinaro, Vehicular Ad hoc Networks (VANET ) Standards, Solutions, and Research., Springer, Torino, 2014.

[5] I. Tan and A. Bahai, "Physical Layer Considerations for Vehicular Communications" in VANET: Vehicular Applications and InterNetworking Technologies, H. Hartenstein and K. Laberteaux, John Wiley \& Sons, pp. 157-217, 2009.

[6] C. Wang and X. Cheng, "Vehicle-to-Vehicle Channel Modeling and Measurements: Recent Advances and Future Challenges", IEEE Communications Magazine, Vol.47, Issues 11, pp. 96-103, 2009.

[7] A. G. Zaji, "Impact of Moving Scatterers on Vehicle-to-Vehicle Narrow-Band Channel Characteristics", IEEE Transactions on Vehicular Technology, Vol. 63, No. 7, pp. 30943106, 2014.

[8] A. S. Akki, "A Statistical Model of Mobile-toMobile Land", IEEE Transactions on Vehicular Technology, Vol. 5, No. 1, pp. 2-7, 1986.

[9] A. S. Akki, "Statistical Properties of Mobile-toMobile Land Communication Channels", IEEE Transactions on Vehicular Technology, Vol. 43, No. 4, pp. 826-831, 1994.

[10] L. Wang and Y. Cheng, "A Statistical Mobileto-Mobile Rician Fading Channel Model",
Vehicular Technology Conference, Vol. 00, No. c, pp. 63-67, 2005.

[11] I. A. Heider, "Improvement of Fading Channel Modeling Performance for Wireless Channel", International Journal of Electrical and Computer Engineering (IJECE), Vol. 8, No. 3, p. 1451, 2018.

[12] S. Yoo, "An improved temporal correlation model for vehicle-to-vehicle channels with moving scatterers", URSI Asia-Pacific Radio Science Conference, p. 2, 2016,.

[13] A. Chelli and P. Matthias, "The Impact of Fixed and Moving Scatterers on the Statistics of MIMO Vehicle-to-Vehicle Channels", In: Proc. of VTC Spring - IEEE 69th Vehicular Technology Conference, pp. 1-6, 2009,

[14] D. Du, X. Zeng, X. Jian, L. Miao, and H. Wang, "Three-Dimensional Vehicle-to-Vehicle Channel Modeling with Multiple Moving Scatterers Derong", Journal Mobile Information Systems, Vol. 2017, p. 14, 2017.

[15] A. Borhani and M. Patzold, "Modeling of Vehicle-to-Vehicle Channels in the Presence of Moving Scatterers", In: Proc. of IEEE Vehicular Technology Conference, 2012.

[16] A. Borhani and M. Patzold, "Correlation and spectral properties of vehicle-to-vehicle channels in the presence of moving scatterers", IEEE Transactions on Vehicular Technology, Vol. 62, No. 9, pp. 4228-4239, 2013.

[17] R. Howard, "The Power Spectral Density", in Principles of Random Signal Analysis and Low Noise Design: The Power Spectral Density and its Applications, Wiley-IEEE Press, pp. 291-294, 2015.

[18] S. Yoo and K. Kim, "Closed-Form Doppler Spectra for Vehicle-to-Vehicle Channels in the Presence of Roadside Scatterers", IEEE Communication. Letter, Vol. 23, No. 1, pp. 136139, 2018.

[19] X. Liang, W. Cao, and X. Zhao, "Doppler Power Spectra for 3D Vehicle-to-Vehicle Channels with Moving Scatterers", IEEE Access, Vol. 6, pp. 42822-42828, 2018.

[20] The Institute of Electrical and Electronics Engineers, IEEE Standard for Information technology Telecommunications and information exchange between systems LAN and MAN Specific requirements - Part 11: Wireless LAN Medium Access Control (MAC) and Physical Layer (PHY) Specifications, IEEE Std 802.11-2016, vol. 2016, pp. 1-3534, 2016. 